\title{
Single-molecule light emission at room temperature on a wide-band-gap semiconductor
}

\author{
H. Yang, ${ }^{1,2,3}$ A. J. Mayne,,${ }^{1, *}$ G. Comtet, ${ }^{1}$ G. Dujardin,,${ }^{1}$ Y. Kuk, ${ }^{4}$ S. Nagarajan, ${ }^{5}$ and A. Gourdon ${ }^{5}$ \\ ${ }^{1}$ Institut des Sciences Moléculaires d'Orsay, Centre Nationale de la Recherche Scientifique (Unité Mixte de Recherche 8214), \\ Bât. 210, Univ Paris Sud, 91405 Orsay, France \\ ${ }^{2}$ IBS Center for Integrated Nanostructure Physics (CINAP), Institute for Basic Science, Sungkyunkwan University, \\ Suwon 440-746, South Korea \\ ${ }^{3}$ Department of Energy Science, Sungkyunkwan University, Suwon 440-746, South Korea \\ ${ }^{4}$ Department of Physics and Astronomy, Seoul National University, Seoul 151-747, South Korea \\ ${ }^{5}$ Centre d'Élaboration de Matériaux et d'Études Structurales - Centre Nationale de la Recherche Scientifique, \\ 29, rue J. Marvig, B.P. 94347, 31055 Toulouse, France
}

(Received 24 March 2014; revised manuscript received 29 August 2014; published 17 September 2014)

\begin{abstract}
Room-temperature light emission from single chemisorbed perylene based molecules adsorbed on silicon carbide ( $\mathrm{SiC}$ ) is probed by scanning tunneling microscopy (STM). A new approach to STM-induced luminescence of a single molecule is explored using a wide-band-gap semiconductor to decouple electronically the molecule from the surface. After molecular adsorption, the lowest unoccupied molecular orbital and the highest occupied molecular orbital (HOMO) both lie within the bulk band gap and below the Fermi level of the substrate. The maximum photon energy of the light emission from the molecule shows a fixed shift of $1.5 \mathrm{eV}$ relative to the maximum energy of the tunnel electrons. This is consistent with the photons being generated by inelastic electron tunneling between the HOMO and the unoccupied electronic states of the STM tip.
\end{abstract}

DOI: 10.1103/PhysRevB.90.125427

PACS number(s): 73.20.At, 73.25.+i, 68.37.Ef, 78.60.Fi

\section{INTRODUCTION}

Electroluminescence of a single molecule using tunnel electrons from scanning tunneling microscopy (STM) is attracting much attention as it provides a unique tool to characterize (i) the chemical structure, (ii) the dynamics of the excited states, and (iii) the charge and energy transfer of a single molecule in interaction with its environment [1].

Photon emission by inelastic electron tunneling (IET) was first observed in tunnel junctions [2,3], where it was recognized that the photon emission originated from the excitation of plasmons at the electrode surface. With the advent of the STM, IET was applied to local atomic-scale excitation [4-7]. On clean surfaces, photon emission by IET with the STM was shown to occur via inelastic transitions between states at the apex of the STM tip and electronic states of the surface [8-10], thus leading to atomic-scale resolution of the photon emission excitation. Subsequently, STM-induced photon emission from molecular layers on surfaces was reported [11-13]. Indeed, submolecular lateral resolution has been achieved, either by probing the molecular vibronic states [14,15] or by using the molecule as a spacer so that the photon emission produced in the nanocavity is modified slightly by the molecule [16-18].

Several STM activated mechanisms have been proposed to explain photon emission from molecular layers, including transitions between the lowest unoccupied and highest occupied molecular orbitals (LUMO $\rightarrow$ HOMO) [19-23] and IET between surface states of the STM tip and molecular vibronic states [22,24]. Similar studies on single molecules instead of molecular layers are very demanding because the molecule needs to be electronically decoupled from the metallic substrate by an insulating layer and must not diffuse across the surface during STM excitation. This has been

*andrew.mayne@u-psud.fr achieved with the LUMO-HOMO optical transition in single molecules $[14,17,18]$, the fluorescence of an anionic molecule $[15,25,26]$, and the radiative transition between electronic states of the STM tip and electronic molecular states [27]. Regardless of the inelastic tunneling mechanism involved, detection of photons in the far field occurs, in most cases, via coupling to the plasmons of the tip-sample nanocavity [21].

In this paper, we explore a new approach for STM excitation of the luminescence of a single molecule by using a wide-bandgap semiconductor to decouple the molecule electronically from the surface. We emphasize that a wide-band-gap bulk semiconductor substrate is markedly different from thin oxide layers [25,28]. In the latter case, energy transfer from the electronically excited molecule to the metallic substrate through the oxide layer can be very efficient and prevent fluorescence from occurring. This type of energy relaxation process does not exist for a bulk wide-band-gap substrate.

Here, we present STM luminescence experiments of the N,N'-bis(1-hexylheptyl)perylene-3,4:9,10-bis (dicarboximide) (DHH-PTCDI) molecule. As we have shown previously, this molecule is chemisorbed on the wide-band-gap $6 H$-SiC $(0001) 3 \times 3$ surface $[29,30]$. Upon molecular adsorption, both the LUMO and HOMO of the molecule are below the Fermi level of the surface and lie within the bulk band gap of $3 \mathrm{eV}$ of the $6 \mathrm{H}$-SiC substrate. In general, electron transport at surfaces is governed by the nature of the interactions between an adsorbed molecule, the surface states, and the bulk states [31]. Here, the strongly bound DHH-PTCDI molecule presents a paradox in that it is isolated electronically from both the bulk and surface states of the $\mathrm{SiC}$ substrate despite being chemisorbed [29,30]. However, the LUMO-HOMO optical transition cannot be observed because the LUMO is completely decoupled from the $\mathrm{SiC}$ surface states. Indeed, the observed electroluminescence of the DHH-PTCDI molecule is assigned to IET between the HOMO of the molecule and the surface states of the STM tip. 


\section{EXPERIMENT}

The STM experiments were performed at room temperature in an ultrahigh vacuum chamber (base pressure $3 \times$ $10^{-11}$ Torr). A highly nitrogen-doped (density $5 \times 10^{19} \mathrm{~cm}^{-3}$ ) $n$-type $6 \mathrm{H}$-SiC(0001) single-crystal wafer was used. After outgassing, the $\mathrm{SiC}$ sample was flashed at $1100^{\circ} \mathrm{C}$ to remove the native oxide, followed by silicon deposition on the surface at $650{ }^{\circ} \mathrm{C}$ for a few minutes [32-34]. Annealing at the same temperature $\left(650^{\circ} \mathrm{C}\right)$ for $10 \mathrm{~min}$ led to a well-reconstructed $3 \times 3$ surface that presented a sharp low energy electron diffraction (LEED) pattern. Silicon deposition is required to produce the $3 \times 3$ reconstruction of the $\mathrm{SiC}(0001)$ surface. This silicon-rich surface is composed of a complete Si adlayer (on top of the bulk) with supplementary Si trimers placed at regular intervals; where each $\mathrm{Si}$ trimer is topped with a $\mathrm{Si}$ adatom to form a pyramid. Only the Si adatoms are visible in the STM images [32]. There are three surface states created by the $3 \times 3$ reconstruction, $U_{1}, S_{1}$, and $S_{2}$ at $+0.8,-0.8$,
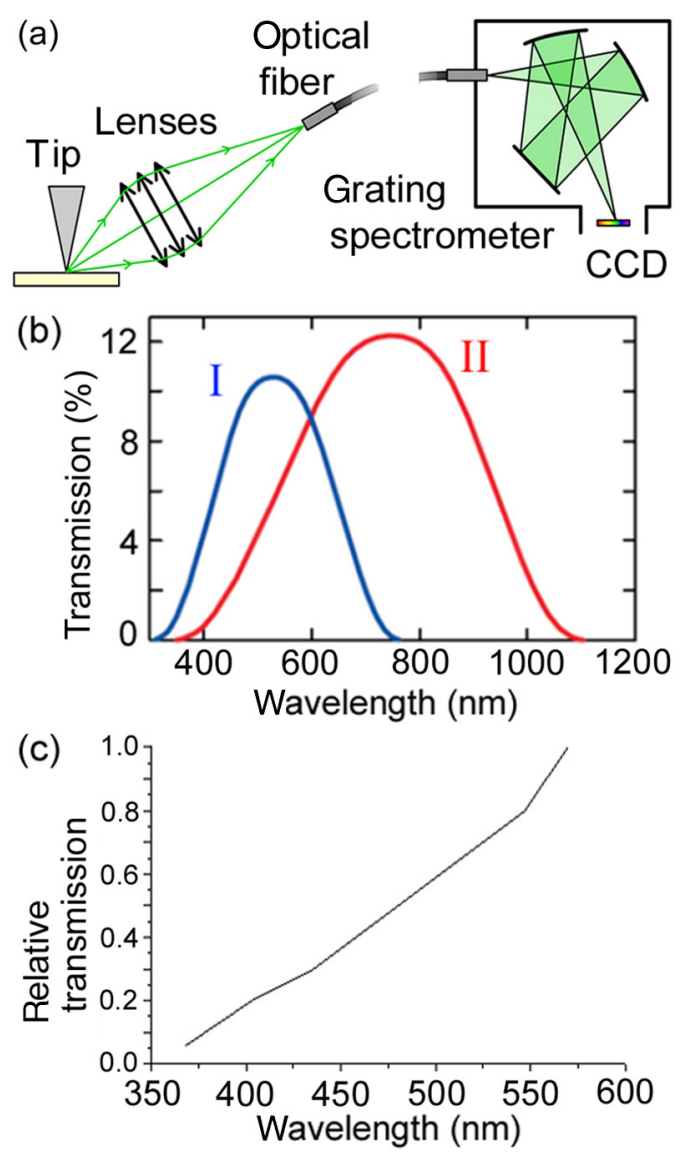

FIG. 1. (Color online) (a) Optical detection apparatus comprise an adjustable in vacuum three-lens system with the focal point $1 \mathrm{~cm}$ beyond the quartz view port [22]. An optical fiber bundle transmits the emitted light to a grating spectrometer, where the light is collected by a liquid- $\mathrm{N}_{2}$-cooled $\mathrm{CCD}$ camera. (b) Optical transmission of the two gratings used in the experiment. (c) Transmission of the optical pathway as a function of wavelength. All spectra were normalized using the total transmission efficiency. Overall the maximum transmission achievable with this optical setup is $12 \%$. and $-1.8 \mathrm{eV}$, respectively; all three are observed in STM experiments [32,33] and photoemission studies [35,36]. The $S_{2}$ state is delocalized over the $\mathrm{Si}$ adalayer between the bulk and the Si pyramids, while the $U_{1}$ and $S_{1}$ states arise from the Mott-Hubbard splitting of the Si adatom dangling bond state into unoccupied $\left(U_{1}\right)$ and half-occupied $\left(S_{1}\right)$ orbitals due to correlation effects. The Fermi level is pinned midway between the $U_{1}$ and $S_{1}$ states $2 \mathrm{eV}$ above the valence band edge and $1 \mathrm{eV}$ below the conduction band edge $[35,36]$.

The detection of photons in the far field occurs via the conversion of the plasmons generated in the tip-sample nanocavity, so electrochemically etched silver (Ag) tips were used because they have a higher conversion efficiency than $\mathrm{W}$ tips. The experimental setup has been described before [32], where the light emitted from the sample under the STM tip was collected at $32^{\circ}$ from the surface by a system composed of three lenses mounted in vacuum [Fig. 1(a)]. The collected light was transmitted through an optical fiber, dispersed by a grating spectrometer, and detected by a liquid nitrogen cooled charge-coupled device (CCD) camera. As shown in Fig. 1(b), the spectra were obtained over a wavelength range from 350 to $1100 \mathrm{~nm}$ (depending on whether grating I or grating II was used). Figure 1(c) shows that the transmission drops towards shorter wavelength. All spectra were normalized using the total transmission efficiency. Overall the maximum transmission achievable with this optical setup is $12 \%$.

The DHH-PTCDI was synthesized by reaction of perylene-3,4,9,10-tetracarboxylic di-anhydride with 1-hexylheptylamine, prepared from 7-tridecanone [37]. Double-pass chromatography (chloroform $/ \mathrm{SiO}_{2}$ ) was used for final purification. The DHH-PTCDI molecules have an optical gap of $2.6 \mathrm{eV}$ [38,39]. They were deposited on the clean $\mathrm{SiC}(0001)-3 \times 3$ surface using a Knudsen cell with a crucible temperature of $200^{\circ} \mathrm{C}$ placed $4 \mathrm{~cm}$ in front of the sample.

\section{RESULTS AND DISCUSSION}

Figure 2 shows filled state STM images of the DHH-PTCDI molecules adsorbed on the $\mathrm{SiC}(0001)-3 \times 3$ surface after two different deposition times of (a) $30 \mathrm{~s}$ and (b) $180 \mathrm{~s}$. The SiC surface is seen as a regular hexagonal array with an adatom spacing of $9.28 \AA$. In Fig. 2(a), there are five molecules in the STM image; each is $14 \AA$ long, composed of double protrusions with an apparent height of $3 \AA$. There are three equivalent orientations of the molecules with respect to the surface corresponding to the threefold structure of the surface [Fig. 2(a)]. A close inspection shows that the long axis of the molecule is slightly offset with respect to the Si adatoms. Prior to adsorption, the molecule is essentially planar in the gas phase. Adsorption occurs through a pseudo-Diels-Alder $12+2$ cycloaddition via two carbonyl oxygen atoms on the same side of the molecule, with two neighboring Si adatoms of the $3 \times$ 3 reconstructed $\mathrm{SiC}$ surface. Adsorption induces significant curvature to the molecule when adsorbed on the SiC surface and strongly modifies the electronic structure; electron transfer from the surface to the molecule populates the LUMO creating a singly occupied molecular orbital (SOMO) just below the Fermi level [29,30]. 

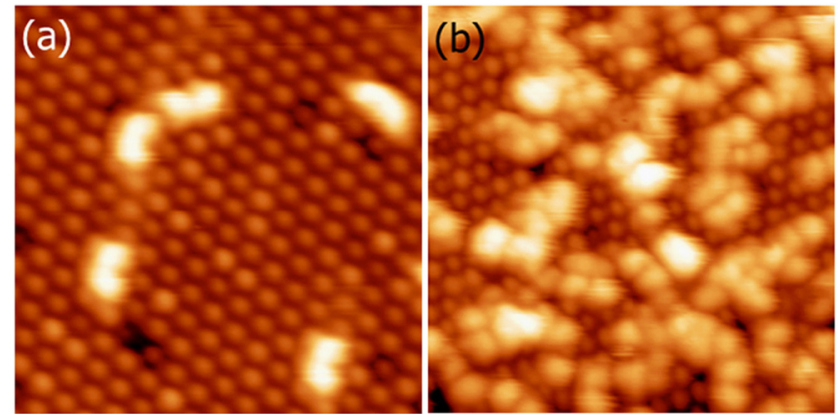

(c)

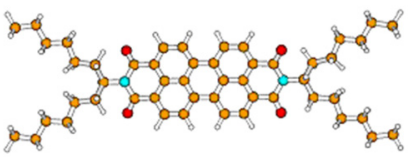

(d)

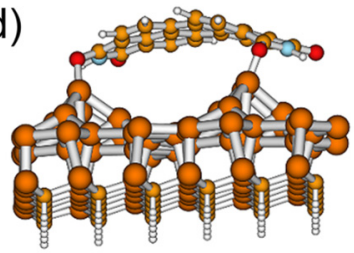

FIG. 2. (Color online) Filled state STM images of the DHHPTCDI molecules adsorbed on the $\mathrm{SiC}(0001)-3 \times 3$ surface after two different deposition times of (a) $30 \mathrm{~s}$ and (b) $180 \mathrm{~s}$, corresponding to 0.1 and 0.9 monolayer. Image conditions: (a) $12 \times 12 \mathrm{~nm},-3 \mathrm{~V}$, $0.3 \mathrm{nA}$; (b) $20 \times 20 \mathrm{~nm},-3 \mathrm{~V}, 0.3 \mathrm{nA}$. (c) Top view of the ball and stick model of the DHH-PTCDI molecule showing the hexylheptyl chains attached to the imide nitrogen atoms. (d) Side view of the ball and stick representation of the DFT calculated adsorption configuration involving bonding via the oxygen atoms of the two carbonyl groups on the same side of the molecule. The alkyl chains are omitted for clarity.

One of the difficulties with room-temperature STM experiments is the presence of thermal drift of the tip with respect to the sample, which is about $2 \AA / m i n$ in our system. Since the size of the DHH-PTCDI molecule is $14 \AA$, the STM tip remains over one molecule for $\sim 2-5 \mathrm{~min}$. To obtain clear luminescence spectra, photons were accumulated for at least $10 \mathrm{~min}$. The drift problem was solved in one of two ways. The first required imaging a high coverage region [approximately 0.9 monolayer of DHH-PTCDI, shown in Fig. 2(b)] successively for the duration of the measuring time for the luminescence spectra, while the second involved taking at least five 4-min-long spectra on a single molecule and adding them (the two methods gave similar results). Luminescence spectra were obtained at different sample voltages $V$ between -3 and $-5 \mathrm{~V}$ and different tunnel currents between 0.5 and $3 \mathrm{nA}$ using a collection time of $1000 \mathrm{~s}$. In our experiment, a negative sample bias is applied implying that the electrons inducing the scattering events pass from the sample to the tip. No spectra were obtained at positive sample bias because above $+3.5 \mathrm{~V}$, electron injection from the STM tip induces molecular displacement across the surface or molecular dissociation.

\section{A. Luminescence of the clean $\mathrm{SiC}$ surface}

Figure 3(a) shows the room-temperature luminescence spectra of the clean $\mathrm{SiC}(0001)-3 \times 3$ surface. On the clean $\mathrm{SiC}$ surface, the light emission spectra are recorded at relatively low tunnel currents of 0.5 and $1 \mathrm{nA}$ in Figs. 3(a) and 3(c), respectively. As a result, $h v_{\max }$, the maximum energy of the emitted photons, varies as a function of the applied
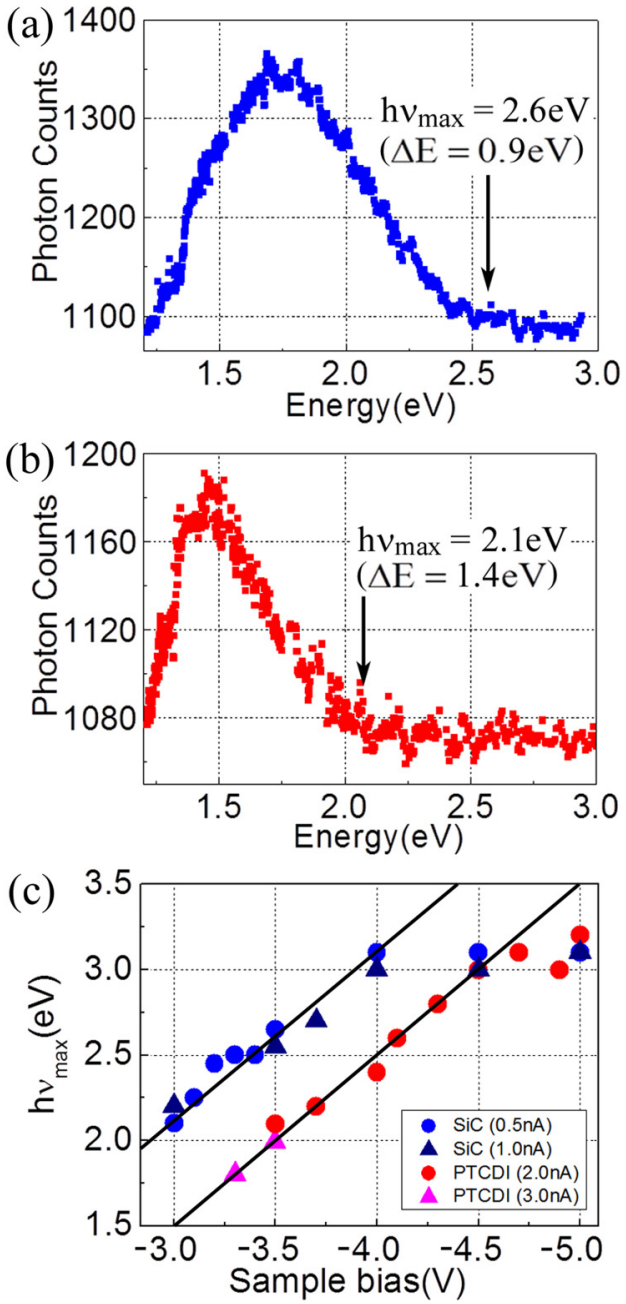

FIG. 3. (Color online) Luminescence spectra taken with a sample bias of $-3.5 \mathrm{~V}$. (a) For a tunnel current $I=1 \mathrm{nA}$, the clean surface shows a difference $\Delta E$ of $0.9 \pm 0.1 \mathrm{eV}$ between the absolute value of the tunnel voltage and the maximum photon energy, with respect to the photon threshold, and (b) for a tunnel current $I=2 \mathrm{nA}$, the molecules show a difference $\Delta E$ of $1.4 \pm 0.1 \mathrm{eV}$. (c) Maximum photon energy $h v_{\max }$ as a function of the sample voltage $V$ measured from the luminescence spectra of the clean $\mathrm{SiC}$ surface (blue circle $=0.5 \mathrm{nA}$ and black triangle $=1.0 \mathrm{nA}$ ) and the molecules (red circle $=2.0 \mathrm{nA}$ and pink triangle $=3.0 \mathrm{nA}$ ). The black lines indicate the average threshold value $e|V|-\Delta E$ of $0.9 \pm 0.15 \mathrm{eV}$ and $1.5 \pm 0.1 \mathrm{eV}$, respectively.

voltage since $h v_{\max }=e|V|-\Delta E$, giving a $\Delta E=0.9 \mathrm{eV}$. The constant difference of $0.9 \pm 0.15 \mathrm{eV}$ between the sample voltage and $h v_{\max }$ suggests that the luminescence is generated by IET between the $S_{1}$ surface state of the clean $\mathrm{SiC}$ and the unoccupied electronic states of the STM tip [Fig. 4(a)]. Indeed in Fig. 4(b), in STM $d I / d V$ spectroscopy the $S_{1}$ surface state is observed at $0.5-0.9 \mathrm{eV}$ below the Fermi level $[29,32]$. No double peak is observed in the emission spectra. In Fig. 3(a), the photon emission through the $S_{1}$ state is favored with respect to the $S_{2}$ state because the $S_{1}$ state is closer to the vacuum level. The transmission probability through the tunnel barrier is higher for the $S_{1}$ state than the $S_{2}$ state because the $S_{2}$ state is lower in energy and faces a wider tunnel barrier. While photon 

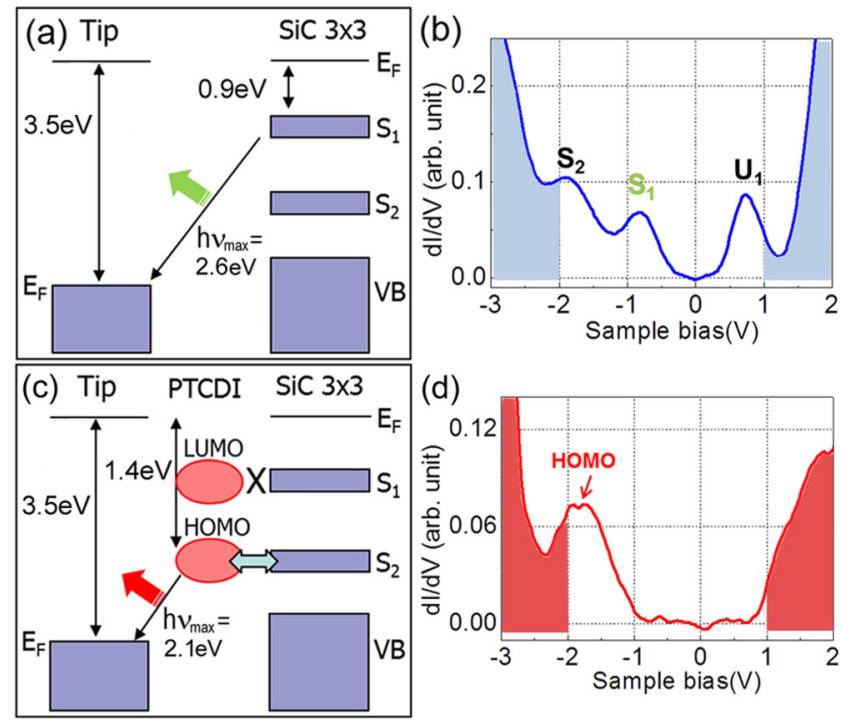

(d)

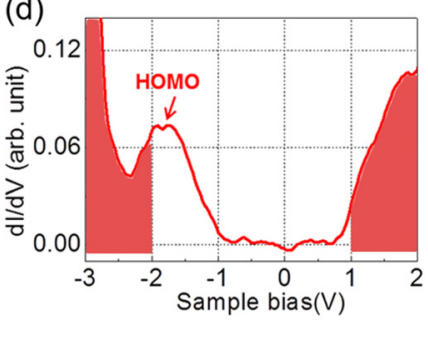

FIG. 4. (Color online) (a) Schematic band diagram of the STM junction on the clean surface, showing photon emission from the $S_{1}$ surface state. (b) Derivative of the $I(V)$ spectroscopy of the clean $\operatorname{SiC}(0001)-3 \times 3$ surface, showing the three surface states $\left(S_{2}, S_{1}\right.$, and $U_{1}$ ) within the bulk band gap. (c) Schematic band diagram of the STM junction on the DHH-PTCDI molecules showing emission from the HOMO. (d) Derivative of the $I(V)$ spectroscopy of the PTCDI molecule adsorbed on the $\mathrm{SiC}(0001)-3 \times 3$ surface, showing a single molecular state (HOMO) within the bulk band gap. In (b) and (d), the shaded areas below $-2 \mathrm{~V}$ and above $+1 \mathrm{~V}$ indicate, respectively, the valence and conduction bands of the $\mathrm{SiC}$ surface. The spectroscopy curves are reproduced from Ref. [29].

emission from the $S_{2}$ state cannot be excluded, it will be hidden below the threshold of the $S_{1}$ emission. Our observations differ from our earlier experiments [32], where photon emission of the clean $\mathrm{SiC}$ surface was observed only from the $S_{2}$ surface state located 1.5-1.8 eV below the Fermi level. In the present experiment, a relatively small tunnel current of $0.5-1 \mathrm{nA}$ was used, whereas higher tunnel currents in the range 3-25 nA were used previously [32]. The change in behavior is due to the high resistance (of the order of $1.0 \mathrm{G} \Omega$ ) of the $S_{1}$ surface state; high tunnel currents (3-25 nA) prevented the observation of any IET through the $S_{1}$ state [32]. The much smaller resistance $(<0.03 \mathrm{G} \Omega)$ of the $S_{2}$ surface state allows IET through the $S_{2}$ surface state with high tunnel currents (3-25 nA), as previously reported [32]. At this point, it is important to mention that we assume a flat band scenario. This is justified for two reasons: (i) the $\mathrm{SiC}$ sample has a high $n$-type doping $\left(5 \times 10^{19} \mathrm{~cm}^{-3}\right)$, and (ii) the Mott-Hubbard splitting of the $\mathrm{Si}$ adatom dangling bond state into $U_{1}$ and $S_{1}$ due to correlation effects pins the Fermi level midway between the $U_{1}$ and $S_{1}$ states.

\section{B. Luminescence of the DHH-PTCDI molecule}

On the DHH-PTCDI molecules, the light emission spectra are recorded at intermediate tunnel current values, i.e., 2-3 nA [Figs. 3(b) and 3(c)]. No spectra could be recorded at tunnel currents lower than $2 \mathrm{nA}$ on the molecule; the light emission intensity is reduced by at least a factor of 3 compared to the clean SiC surface. The maximum energy of photons increases linearly as the tunneling voltage $V$ is increased up to about $-4.5 \mathrm{eV}$, according to $h v_{\max }=e|V|-\Delta E$. For higher voltages, the maximum photon energy remains the same due a reduction in transmission of the optical system above $3.2 \mathrm{eV}(\sim 400 \mathrm{~nm})$. The plasmon response of the Ag tip might also contribute to this saturation of the maximum energy of the photons. The energy difference $(\Delta E$ ) for molecular luminescence between the absolute value of the tunneling voltage and the maximum photon energy is $1.5 \pm 0.1 \mathrm{eV}$ in the tunneling regime and for sample biases between $-3.3 \mathrm{eV}$ and $-4.5 \mathrm{eV}$. The linear variation of $h v_{\max }$ as a function of the sample voltage shows that the luminescence of PTCDI on $\mathrm{SiC}$ is not produced by an interorbital transition (e.g. LUMO $\rightarrow \mathrm{HOMO}$ ), since $h v_{\max }$ would not vary with the sample voltage. Instead, the constant difference of $1.5 \pm 0.1 \mathrm{eV}$ between the sample voltage and $h v_{\max }$ suggests that the luminescence is from IET between the molecular HOMO and the unoccupied electronic states of the STM tip, similar to the observation by Lutz et al. [28]. The HOMO lies 1.5-1.8 eV below the Fermi level of the SiC substrate [29,32], corresponding to the measured $\Delta E$ value of $1.5 \mathrm{eV}$. In passing, two things should be noted: first the scanning tunneling spectroscopy (STS) experiments were performed at room temperature and second the HOMO state was partially decoupled from the surface (see discussion of the luminescence mechanism below). Both a partially decoupled HOMO and room-temperature experiments will naturally give a broad peak.

\section{Luminescence mechanism}

The luminescence mechanism can be understood from the schematic of the light emission presented in Fig. 4(c) and the STM $d I / d V$ spectroscopy of the adsorbed PTCDI molecule in Fig. 4(d). Due to charge transfer from the substrate to the molecule, the LUMO is shifted below the Fermi level. Density functional theory (DFT) calculations give a value of $-0.2 \pm$ $0.4 \mathrm{eV}$ for the position of the LUMO that is not visible in the STS. Given that these orbitals and surface states have a certain width of around $0.6-0.8 \mathrm{eV}$, one can consider that the LUMO and $S_{1}$ are in the same energy window. On the other hand, the HOMO has an energy close to that of the $S_{2}$ surface state [29]. Compared to molecules adsorbed on a metallic substrate [28], IET from the LUMO and/or HOMO is more difficult to achieve in the case of a molecule adsorbed on a wide-band-gap surface, such as SiC, since both the LUMO and HOMO lie within the bulk band gap $(3 \mathrm{eV})$ of the $\mathrm{SiC}$ substrate. In this case, the STM current must travel across the surface through the surface states $\left(S_{1}\right.$ and $\left.S_{2}\right)$ before it can tunnel inelastically through the LUMO and/or the HOMO. In fact, the electronic interaction between the LUMO and the $S_{1}$ surface state is significantly reduced compared to the interaction between the HOMO and the $S_{2}$ surface state [29]. Upon adsorption, the PTCDI molecule forms two new Si-O bonds between the carbonyl groups of the molecule and the $\mathrm{Si}$-adatom of the $\mathrm{SiC}$ surface. As we showed in the DFT calculations previously [29], electron density is found within the energy window from -3.7 to $-4.3 \mathrm{eV}$, indicating the presence of the $\mathrm{Si}-\mathrm{O}$ bonds. Before 
adsorption, the DFT calculations also clearly showed electron density associated with the $S_{1}$ state $(-0.3 \mathrm{eV}$ below the Fermi level). After adsorption, the LUMO is displaced below the Fermi level as a result of accepting an extra electron and is observed in the same energy window as the $S_{1}$ state before adsorption, only there is now no electron density associated with the $S_{1}$ state. The $\pi$-system of the PTCDI molecule is relatively unchanged, even though the molecule is no longer planar [29,30]. The absence of electron density will reduce the conductivity, effectively decoupling the LUMO from the surface [marked by the X in Fig. 4(c)]. As a result, the LUMO is completely invisible in the conductance spectra-from the $d I / d V$ spectra the LUMO-substrate resistance is estimated at > $30 \mathrm{G} \Omega$; no current passes through the LUMO. The interaction of the LUMO with the delocalized $S_{2}$ state will be weak for the same reasons: (i) the energy difference is around $1.5 \mathrm{eV}$ and (ii), the LUMO is $5 \AA$ above the $S_{2}$ state. On the other hand, after adsorption of the molecule the $S_{2}$ surface state is still present underneath the molecule; the HOMO-substrate resistance was estimated from $I(Z)$ spectroscopy at around 2 $\mathrm{G} \Omega$. In this case, the current can pass through the HOMO. In addition, the tip is roughly $10 \AA$ above the $S_{2}$ adlayer state when it is over the molecule. This will reduce significantly any emission from the $S_{2}$ state. It follows that passing the current across the sample through the $S_{1}$ state and then tunneling (elastically or inelastically) through the LUMO is much less probable than passing the current through the $S_{2}$ surface state and tunneling through the HOMO. Thus, the observed photon emission is only possible by IET from the HOMO to the unoccupied states of the STM tip [red arrow in Fig. 4(c)]. The probability of other electronic mechanisms involving the LUMO are significantly reduced, in particular, the LUMO $\rightarrow$ HOMO transition is not observed.

\section{CONCLUSION}

Wide-band-gap materials, such as $\mathrm{SiC}$, having an energy gap $(3 \mathrm{eV})$ that is larger than the HOMO-LUMO gap of molecule, offer interesting perspectives to achieve STM electroluminescence of a single molecule. In the particular case studied here of a DHH-PTCDI molecule adsorbed on a $6 H$-SiC(0001)-3 $\times 3$ surface, the LUMO and HOMO of the molecule both lie within the bulk band gap and are below the Fermi level of the substrate. Nevertheless, even with this electronic configuration of the molecule-surface system, the photon emission process is complex since it could occur from (i) IET between the LUMO and the unoccupied states of the STM tip, (ii) IET between the HOMO and the unoccupied states of the STM tip, or (iii) the LUMO $\rightarrow$ HOMO transition. The balance between these three photon emission processes depends on the strength of the electronic couplings between the LUMO and HOMO of the molecule and the $S_{1}$ and $S_{2}$ surface states as well as the resistance of the $S_{1}$ and $S_{2}$ surface states across the surface. Due to the high resistance of $S_{1}$ relative to $S_{2}$ and the poor electronic coupling between the LUMO and $S_{1}$ (relative to the electronic coupling between the HOMO and $S_{2}$ ), the observed photon emission is dominated by the inelastic electron transition between the HOMO and the unoccupied electronic states of the STM tip.

\section{ACKNOWLEDGMENTS}

This work is supported by the Institute for Basic Science in South Korea, the French National Research Agency (Molsic ANR-08-NANO-030), the Hubert Curien Program (Star 21424ZA), and the Advanced Thematic Research Network (2008-024T).
[1] F. Rossel, M. Pivetta, and W.-D. Schneider, Surf. Sci. Rep. 65, 129 (2010).

[2] J. Lambe and S. L. McCarthy, Phys. Rev. Lett. 37, 923 (1976).

[3] P. Dawson, D. G. Walmsley, H. A. Quinn, and A. J. L. Ferguson, Phys. Rev. B 30, 3164 (1984).

[4] J. K. Gimzewski, J. K. Sass, R. R. Schlittler, and J. Schott, Europhys. Lett. 8, 435 (1989).

[5] P. Johansson and R. Monreal, Z. Phys. B 84, 269 (1991).

[6] R. Berndt, J. K. Gimzewski, and P. Johansson, Phys. Rev. Lett. 67, 3796 (1991).

[7] A. J. Mayne, G. Dujardin, G. Comtet, and D. Riedel, Chem. Rev. 106, 4355 (2006).

[8] C. Thirstrup, M. Sakurai, K. Stokbro, and M. Aono, Phys. Rev. Lett. 82, 1241 (1999).

[9] M. Sakurai, C. Thirstrup, and M. Aono, Phys. Rev. Lett. 93, 046102 (2004).

[10] G. Schull, M. Becker, and R. Berndt, Phys. Rev. Lett. 101, 136801 (2008).

[11] R. Berndt, R. Gaisch, J. K. Gimzewksi, B. Reihl, R. R. Schlittler, W.-D. Schneider, and M. Tschudy, Science 262, 1425 (1993).

[12] D. Fujita, T. Ohgi, W.-L. Deng, K. Ishige, T. Okamato, S. Yokoyama, K. Kamikado, and S. Mashiko, Surf. Sci. 454456, 1021 (2000).
[13] S. Mühlenberend, N. L. Schneider, M. Gruyters, and R. Berndt, Appl. Phys. Lett. 101, 203107 (2012).

[14] C. Chen, P. Chu, C. A. Bobisch, D. L. Mills, and W. Ho, Phys. Rev. Lett. 105, 217402 (2010).

[15] J. Lee, S. M. Perdue, A. Rodriguez Perez, and V. A. Apkarian, ACS Nano 8, 54 (2014).

[16] G. Hoffmann, L. Libioulle, and R. Berndt, Phys. Rev. B 65, 212107 (2002).

[17] S. E. Zhu, Y. M. Kuang, F. Geng, J. Z. Zhu, C. Z. Wang, Y. J. Yu, Y. Luo, Y. Xiao, K. Q. Liu, Q. S. Meng, L. Zhang, S. Jiang, Y. Zhang, G. W. Wang, Z. C. Dong, and J. G. Hou, J. Am. Chem. Soc. 135, 15794 (2013).

[18] G. Reecht, F. Scheurer, V. Speisser, Y. J. Dappe, F. Mathevet, and G. Schull, Phys. Rev. Lett. 112, 047403 (2014).

[19] W. Deng, D. Fujita, T. Ohgi, S. Yokoyama, K. Kamikado, and S. Mashiko, J. Chem. Phys. 117, 4995 (2002).

[20] E. Ćavar, M.-C. Blüm, M. Pivetta, F. Patthey, M. Chergui, and W.-D. Schneider, Phys. Rev. Lett. 95, 196102 (2005).

[21] Z. C. Dong, X. L. Zhang, H. Y. Gao, Y. Luo, C. Zhang, L. G. Chen, R. Zhang, X. Tao, Y. Zhang, J. L. Yang, and J. G. Hou, Nat. Photonics 4, 50 (2010).

[22] F. Geng, Y. Zhang, Y. Yu, Y. Kuang, Y. Liao, Z. Dong, and J. Hou, Opt. Express 20, 26725 (2012). 
[23] N. L. Schneider and R. Berndt, Phys. Rev. B 86, 035445 (2012).

[24] N. L. Schneider, F. Matino, G. Schull, S. Gabutti, M. Mayor, and R. Berndt, Phys. Rev. B 84, 153403 (2011).

[25] X. H. Qiu, G. V. Nazin, and W. Ho, Science 299, 542 (2003).

[26] S. W. Wu, G. V. Nazin, and W. Ho, Phys. Rev. B 77, 205430 (2008).

[27] T. Lutz, C. Grosse, C. Dette, A. Kabakchiev, F. Schramm, M. Ruben, R. Gutzler, K. Kuhnke, U. Schlickum, and K. Kern, Nano Lett. 13, 2846 (2013).

[28] N. Nilius, N. Ernst, and H.-J. Freund, Phys. Rev. Lett. 84, 3994 (2000).

[29] H. Yang, O. Boudrioua, A. J. Mayne, G. Comtet, G. Dujardin, Y. Kuk, Ph. Sonnet, L. Stauffer, S. Nagarajan, and A. Gourdon, Chem. Phys. Phys. Chem. 14, 1700 (2012).

[30] O. Boudrioua, H. Yang, Ph. Sonnet, L. Stauffer, A. J. Mayne, G. Comtet, G. Dujardin, Y. Kuk, S. Nagarajan, A. Gourdon, and E. Duverger, Phys. Rev. B 85, 035423 (2012).
[31] G. Dujardin, A. J. Mayne, and F. Rose, Phys. Rev. Lett. 89, 036802 (2002).

[32] G. Baffou, A. J. Mayne, G. Comtet, and G. Dujardin, Phys. Rev. B 77, 165320 (2008).

[33] F. Amy, H. Enriquez, P. Soukiassian, C. Brylinski, A. Mayne, and G. Dujardin, Appl. Phys. Lett. 79, 767 (2001).

[34] F. Amy, H. Enriquez, P. Soukiassian, P.-F. Storino, Y. J. Chabal, A. J. Mayne, G. Dujardin, Y. K. Hwu, and C. Brylinski, Phys. Rev. Lett. 86, 4342 (2001).

[35] J. Schardt, J. Bernhardt, U. Starke, and K. Heinz, Phys. Rev. B 62, 10335 (2000).

[36] L. S. O. Johansson, L. Duda, M. Laurenzis, M. Krieftewirth, and B. Reihl, Surf. Sci. 445, 109 (2000).

[37] M. W. Holman, R. C. Liu, and D. M. Adams, J. Am. Chem. Soc. 125, 12649 (2003).

[38] A. J. Ferguson and T. S. Jones, J. Phys. Chem. B 110, 6891 (2006).

[39] H. Méndez, I. Thurzo, and D. R. T. Zahn, Phys. Rev. B 75, 045321 (2007). 